\title{
AERODYNAMIC THRUST CHARACTERISTICS AND PERFORMANCE VALUATIONS FOR AN AEROSPIKE NOZZLE- REVIEW
}

\author{
Ankit Kumar Mishra \\ Department of Aerospace engineering \\ Lovely Professional University, Phagwara, Punjab, India
}

\author{
Vinit Goswami \\ Department of Aerospace engineering \\ Lovely Professional University, Phagwara, Punjab, India
}

\author{
Priyanka Sarma \\ Department of Aerospace engineering \\ Lovely Professional University, Phagwara, Punjab, India
}

\begin{abstract}
Aerospike nozzles are now-a-days one of the most emerging topics for research and development work leading for the futuristic goals of an aerospace industries and organizations. They are indulging in a class of elevation balancing nozzle engines. These nozzle engines are basically constructed for single-stage-to-orbit (SSTO) as well as foremost engine for space vehicles. These nozzles are about $\mathbf{7 5 \%}$ shorter than the standard nozzle for similar expansion ratios. Aerodynamic thrust variation and performances play a key role in estimating forces along with the injected flow and their characteristics. This review paper deals with the aerodynamics characterization, its properties at different conditions in addition with the performance analysis of the aerospike nozzle.
\end{abstract}

Keywords - Aerodynamic thrust, Review, Secondary flow, Truncated length.

\section{INTRODUCTION}

The advancement in propulsion systems potentially reflects the spacecraft's mass production. Mass reduction is majorly affected in case of planetary landing missions as well as for high altitude propulsive systems [1]. Propulsion systems suited with conventional nozzles shows the efficiency loss of $15 \%$ which are operating from sea to vacuum conditions. For such cases, the aerospike nozzles trap over $50 \%$ of losses than in conventional nozzles. The characteristics of trap are due to ability of compensation at different altitude as well as at launching and climbing phases. The expansion flows are selfadjustable at altitudes than the plotted altitudes which is responsible for improvement in thrust and its corresponding coefficients. At high altitude, the operational performance of aerospike nozzles is probably similar to that of conventional nozzles. For the condition of exit pressure equals the ambient pressure, the expansion is completely optimistic or fully expanded. At lower altitudes, the exit pressure is lower than the ambient pressure for which the flow is said to be over expanded because of incomplete expansion and the jet exit velocity of nozzle is comparably optimum. The atmospheric pressure represents out the function of altitude, as optimum efficiency is limited only to a single level for a fixed nozzle in addition with the pressure losses that are exposed at variant altitudes. For supersonic nozzle, flow separation found due to stagnation of boundary layer.

\section{LITERATURE REVIEW}

Eilers et.al (2010) investigated the systematic and experimental results of thrust vectoring for an aerospike nozzle. The evaluation of thrust vectoring is done with the help of four injected port positioned at $50 \%$ of total nozzle length. Two-dimensional method of characteristics is taken in account for arranging the system as well as estimating the nozzle contours. CFD analysis as well as in-situ investigation were carried for the injected port system for which the flow throbs produce side forces. The estimation of side forces was $1.5 \%$ of total thrust. The results obtained show the CFD solutions to be of inviscid, axis-symmetric incompressible flows in injected ports for thrust vectoring. The complex geometry of apparatus and instrumentation were calibrated and operated to study the forces and moments generated during the experimental work. An uncertainty testing for cold flow optimization that defines for the static modelling considered as the objective of the work. The total of four tests were conducted for the aerospike nozzle out of which the first two tests' results in shakedown in order to verify the simulating data and its functionality. The other two tests were to analyse the flow vibrates that originates the side forces and moment with a higher degree of reliability [1].

Propst et.al (2014) demonstrated the thrust vectoring for small aerospike nozzle that is capable of thrust generation at $3 \mathrm{kN}$ and expansion ratio of 2.94 . The numerical optimization 
and analysis were done using ANSYS software for two dimensional equations for which the truncation of spike varies from $0-75 \%$. The two different injected sites were chosen as $60 \%$ and $90 \%$ of the truncated length along with fluctuating mass flow rates. This experimental work shows the secondary injected system to be viable and best suited for thrust vectoring. The application of one-dimensional isentropic flow is found to be useful for generating nozzle geometry. CFD analysis played a major role in estimating the aerodynamic thrust vectoring for nozzle outline. The study for plug nozzle is examined for secondary injector flow for specific mass flow rates at different locations. A maximum navigation force is estimated for nozzle formations with inoculation site at $90 \%$ of thorn length [2].

Ajith et.al (2016) presented the pressure estimation for the aerospike nozzle for thrust vector controlling. The regular optimization of rocket engine with shortened nozzles for thrust vectoring at different altitudes without the usage of subordinate jets. The numerical simulations are done with the three-dimensional density-based solver on basis of shear-stress transport (SST) model. The thrust vectoring has achieved the maximum mass flow rate through thorn of the nozzle. The parametric analysis for aerospike nozzle results in $42.99 \%$ of the spike tip area for lower elevations in order to provide better efficiency. The better performance of this aerospike nozzle also results out in desirable flight altitude conditions for key space missions improving the load capacity of the launch vehicles. The ambient as well as operating pressure played an important role for the simulation and optimizing of the aerospike nozzle. The numerical study done in this paper direct towards the 3D modelling and transforms the further meets required for deep space missions [3].

Nair et.al (2017) studied the computational analysis for the aerospike nozzle at different distances as 40, 50 and $60 \%$ to spike length. The computational analysis is based on spalartallamus model along with the steady as well as Navier stokes equations. The results for the flow behaviour, performance and its features are achieved at initial conditions of experimental work. The flow caused by the shock boundary layer separation for a low NPR results in formation of lateral forces. The flow attached with the truncated nozzles reduces the length which affects the thrust production from open area to closer ones [4].

Reza et.al (2017) examined an aerospike nozzle based on internal as well as external factors depending upon altitude and other factors. The MATLAB and method of characteristics are two important consideration used for designing the nozzle for which the simulation is further done by computational fluid dynamics (CFD) in ANSYS (Fluent). The fuels considered for the experimental work are- Papi 94 and HTPB in addition with $\mathrm{N}_{2} \mathrm{O}$ used as an oxidant for combustion process. The results of Mach number also showed advantages of an aerospike nozzle that is best suited over conservative nozzles. The evaluated truncation length lies at $45 \%$ of the total in order to provide the Mach number at 2.6.
The reference of Mach number 3 is taken in consideration for all the nozzle outlines to get the better implementation and efficiency. The comparative results of exit Mach number pampered with FLUENT and egress Mach number with code is found to be similar as for the truncation [5].

Dumitrescu et.al (2018) evaluated the performances of three different shortened lengths for the aerospike nozzle. The three different lengths are as- $40 \%, 50 \%$ and $60 \%$ in overall. For expansion process, forfeiture of thrust is observed for shortening length whereas it is neglected in case of under expanded flow process. The CFD analysis gives an ideal result that deals with altitude and performance of nozzle for the propulsion systems. The results were evaluated by varying the inlet conditions such as pressure, temperature that led to growth in velocity. Based on above results it is concluded that maximum performance is observed for $40 \%$ of the truncated length [6].

Schwer et.al (2018) studied the pressure features of an aerospike nozzle for a detonating engine. A sequence of continuous rotating detonation combustion chamber (RDC) tests was performed for which the equivalence ratios vary from 1.024 to 0.655 provided with bay conditions for nozzle optimization. The results obtained show the shock wave structure that leaps back nearby extended portion of nozzle for steady flow conditions. The numerical as well as the RDE calculations shows the unique characteristics of an aerospike nozzle. The unsteady cause in RDC flow behaviour is similar to that of stable influx behaviour that spreads through stream which explains the accelerating move up of aerospike nozzle [7].

Kurita et.al (2020) investigated the three- dimensional replication for the detonating engine attached with an aerospike nozzle. The main objective behind this simulation is to simplify the propulsive performance for different outlines of nozzles as - open/obstruct and whole/shortened. The equivalence ratio for the nozzle varies from 0.9-1.4 for simulation. The simulating results describes that the specific impulse and features effectiveness for choked flow is around 4-6\% sophisticated than those of open nozzle. The difference in results for thrust presentation between complete and shortened nozzle is smaller than $2 \%$ with others. The results for the detonating velocities for open and dismayed flow are about $96-98 \%$ and $97-99 \%$ with velocity, respectively. The overall nozzle devotes around $1-2 \%$ of total thrust performance which increases as compared to shortened nozzle taken in consideration for the experimental work [8].

\section{DISCUSSION AND ANALYSIS}

\section{A. Method of characteristics}

The method of characteristics solutions are one of the important considerations in order to determine the aerodynamic thrust vectoring of aerospike nozzle. The different operating conditions were set to evaluate the 
parameters for method of characteristics. The mean sea level (MSL) altitude for operating condition is set to $1360 \mathrm{~m}$ whereas for the design sustainability it is taken as $4210 \mathrm{~m}$ at exit lines. The spike truncation ratio $\left(\eta_{\text {trun }}\right)$ considered as $57 \%$ of whole length for altitude of operating condition i.e., $1360 \mathrm{~m}$ at a pressure of $775 \mathrm{kPa}$ and chamber temperature of $300 \mathrm{~K}$ expecting the thrust progression of $454 \mathrm{~N}$ and mass flow rate value of $1.02 \mathrm{~kg} / \mathrm{s}$. A little variation in chamber temperature at $255 \mathrm{~K}$ and absolute mass flow of $1.11 \mathrm{~kg} / \mathrm{s}$ results the thrust to be around $422.7 \mathrm{~N}$ for same nozzle. For design conditions i.e., at altitude of $4210 \mathrm{~m}$ for $\eta_{\text {trun }}$ of $57 \%$ the thrust level obtained is $478 \mathrm{~N}$ and the spike truncation ratio of $75 \%$ the thrust level increases to $481.3 \mathrm{~N}$. From these results it is clear that the performance of the nozzle decreases by $6 \%$ than the earlier estimation. Due to reduce in the pressure about $660 \mathrm{kPa}$ the thrust and mass flow rate reduces by $20 \%$ in all (Eilers et.al 2010). An image for an aerospike nozzle operating at $\mathrm{Po}=775$ $\mathrm{kPa}$ is displayed below-

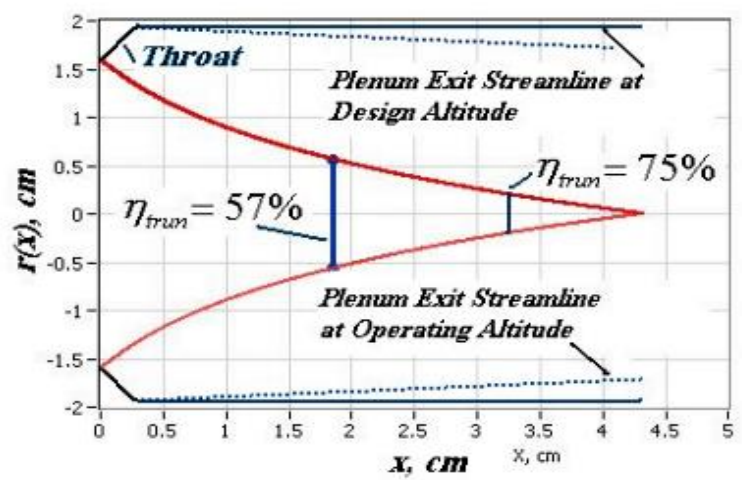

Figure 1. Contour variation from method of characteristics [1] The method of characteristics is used for the calculations of boundary contours and characteristics lines. It is obtained that the efficiency of nozzle rises with characteristics lines which results in smoother flow lines. The contour of nozzle is estimated by using $\mathrm{C}+$ and $\mathrm{C}$ - characteristics for which the first point is considered randomly and the last point is represented on $\mathrm{C}+$ line. The calculation will be carried till the radial component of regressive features is superior to outspread component of stream function (Reza et. al 2017). The geometry for the contour plot is displayed below-

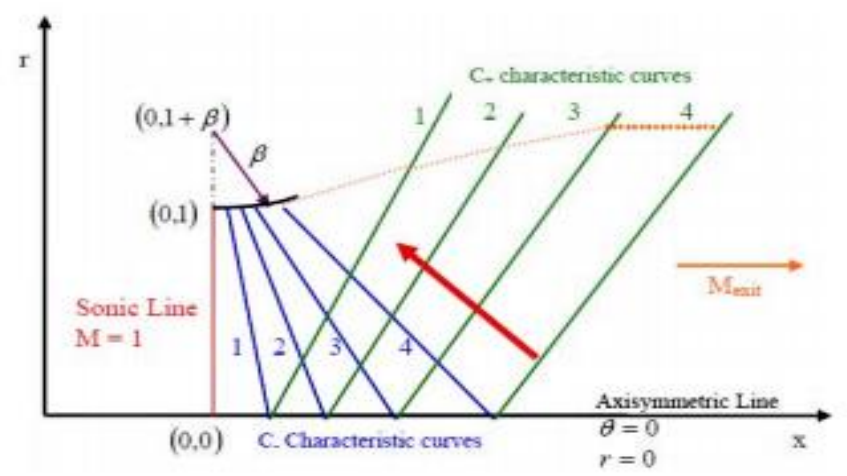

Figure 2. Geometry for contour of nozzle [5]

\section{B. CFD Analysis}

The CFD characteristics considered for simulation and its design requirement as inlet temperature at $300 \mathrm{~K}$ and chamber pressure of $775 \mathrm{kPa}$. The divergent results for CFD and method of characteristics steps down the pressure for throat region for which the flow angle is idealized and characteristics line is responsible for the breakage of codes. The plot for the contour configuration for Mach number is displayed below-

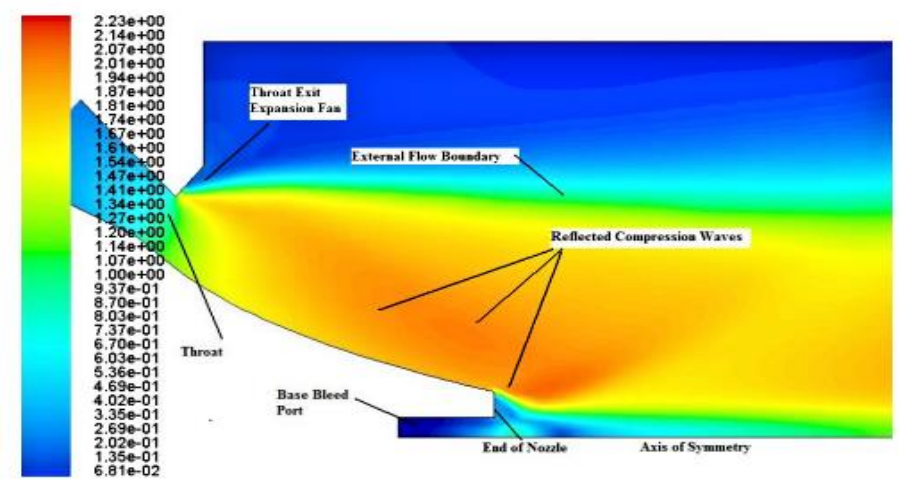

Figure 3. CFD based Mach number contour formations for aerospike nozzle [1].

The compressible waves are shown undoubtedly which is also underlying the aerospike nozzle surface to be $57 \%$ of all. This yield results in decreasing of the pressure value that is similar to contour of space pusher. The CFD results for injected flow for aerospike surface has displayed below-
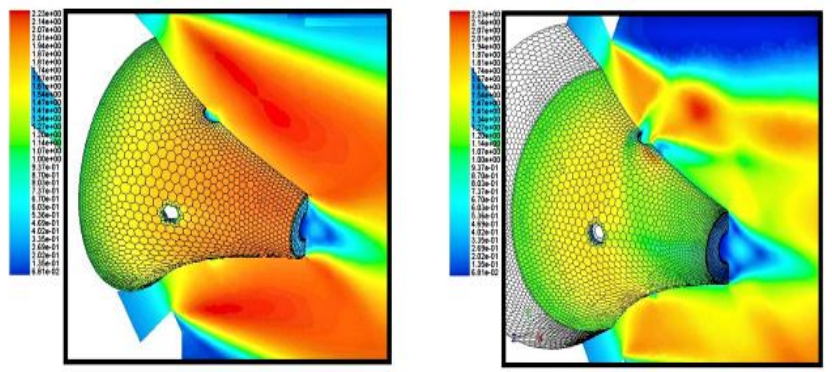

Figure 4. Mach contours (a) without flow injection, (b) with flow injection [1].

These simulations were contoured at down pressure of 775 $\mathrm{kPa}$ for gases to be injected in topmost flow region. The thrust force generation in conventional bell nozzle is completely different from that generated on aerospike surface. At downstream of injected interface, a low pressure region is generated by expansion of fluid and a higher region is formed by extension of bow shockwaves. Due to this the local pressure value increases which results in amplification of thrust created for secondary flow. The convex nature of aerospike surface shrinks the injection port leaving out short pressure region [1]. The CFD simulations for fluctuating spike trunk is calculated followed by up linear as well as non-linear equations in addition with flow characteristics for different 
conditions. The nozzle truncation from 0 to $75 \%$ is been divided into four different segments showing out the dissimilarity in truncation. The CFD results for 0, 25, 50 and $75 \%$ spike truncation is shown below-

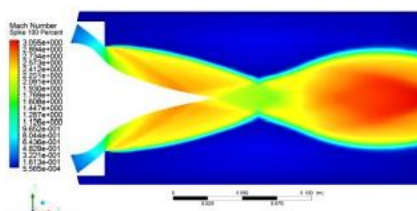

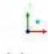

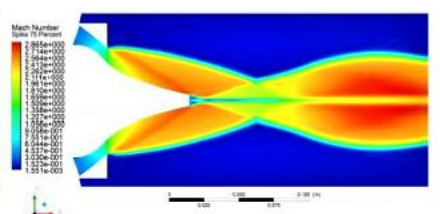

$$
\text { (a) }
$$

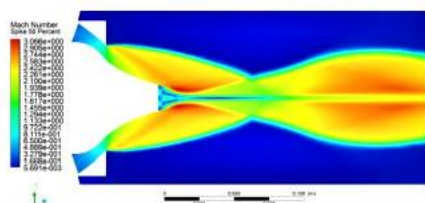

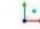

$$
-
$$

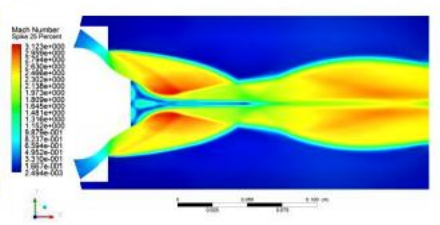

(d)

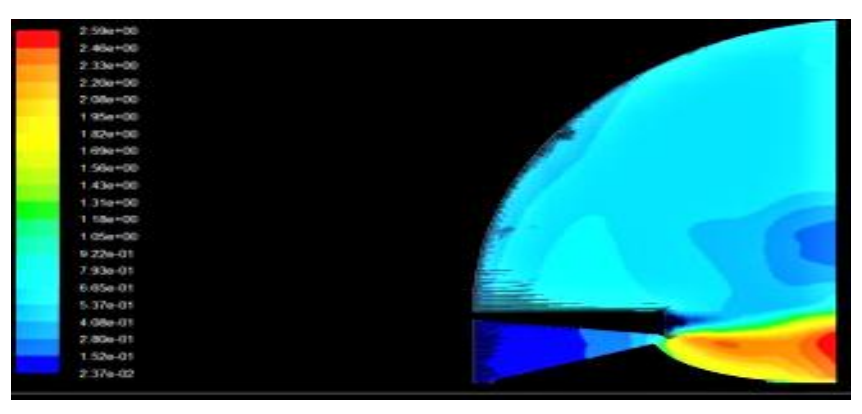

Figure 4. Spike truncations for (a) $0 \%$, (b) $25 \%$, (c) $50 \%$ and (d) $75 \%$ [2].

The distribution shown above is applicable for over expanded flow that is a purpose of Mach number reflecting as onedimensional isentropic flow. The simulation tests for aerospike nozzles are carried out for high altitude regions which are designed to be specific at ground level. The higher percentage in truncation leads to the formation of higher amount of pressure for shock interactions. Meanwhile, the size of close area also increases for base tip portion [2]. The CFD simulations for two different nozzles were performed at altered conditions using MATLAB code in order to attain the chosen Mach number. The medium is considered to be a mixture of gases and air and the boundary conditions as inlet, outlet and wall. The results for different truncation length of CFD analysis are displayed below -

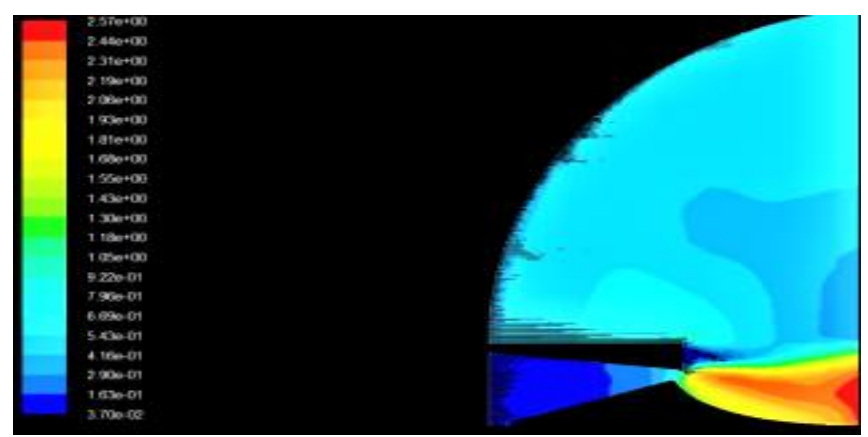

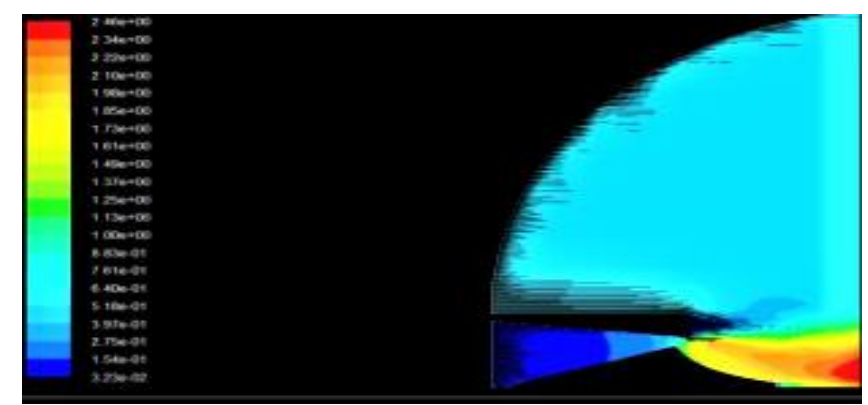

Figure 5. CFD Analysis of nozzle at truncation of (a) 20\%, (b) $40 \%$ and (c) $48 \%$ [5]

The above contour plots shown are at $20 \%, 40 \%$ and $48 \%$ of truncation length for which the extreme truncation is seen for $48 \%$ afterwards it decreases [5].

\section{Parametric Evaluations}

Injected portion for $90 \%$ of point length

As with increase in inoculated flow the strength for shockwaves increases for which the shock wave extends along the free boundary regions. The free-stream flow is focused towards unified solidity shock beside the spike. In overall, the exhaust gas flow gets enlarges for an increasing mediocre mass flow. The shiny point for bow shock results in cuddling of primary flow in one-sided direction. These secondary flows also resists the shear layers for exhaust gas flow in the nozzle. The Mach number plot for injected flow for $90 \%$ of length is shown below-

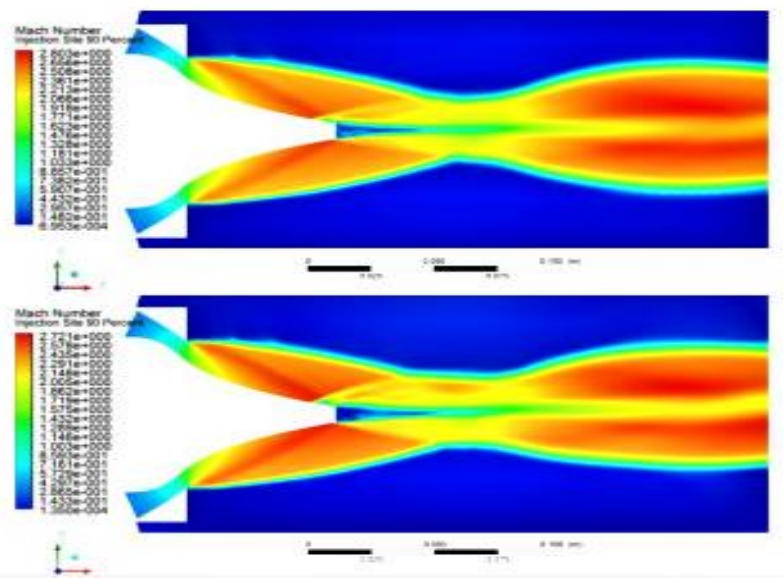


Figure 6. Injected flow for 90\% of spike length [2].

\section{Injected portion for $60 \%$ of point length}

When the injected portion is taken near to nozzle section, the shock wave interacts out with primary flow. As of closer, the shock relates with shear layer of flow at minimum mass flow. With an increase in injected mass, flow sequence varies along strength of shape. The expanded region reaches high pressure zone for which generation of new shock wave occurs opposing free-stream at boundary layer [2]. The injected portion for $60 \%$ of truncated length is displayed below-

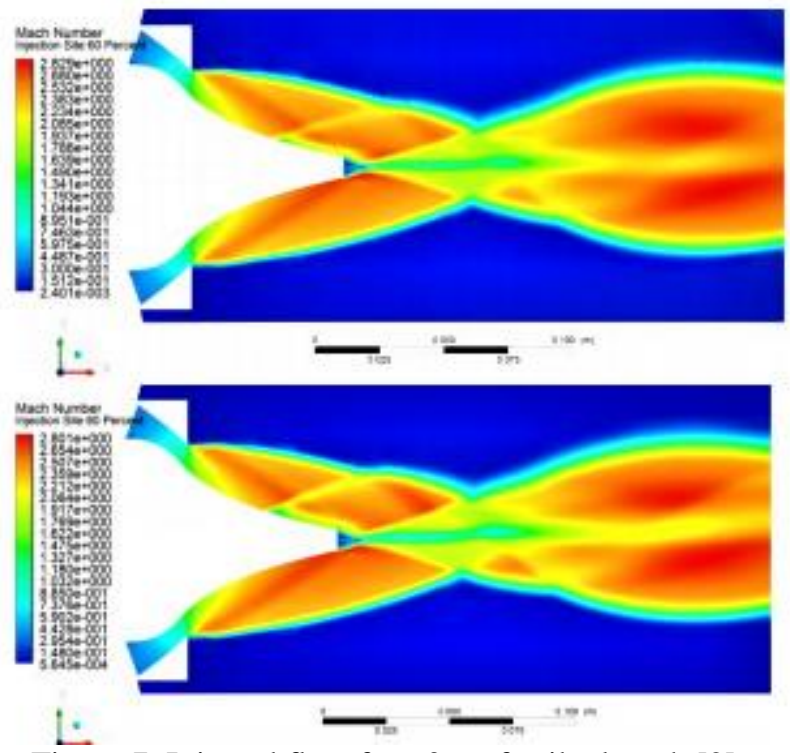

Figure 7. Injected flow for $60 \%$ of spike length [2].

\section{Base pressure ratio for $25 \%$ spike length}

The waking region becomes larger as NPR value increases for 2-D nozzle at different boundary conditions. The extent of waking area is nearly alike for energy NPR. The recent outline for spike led to appearance of shock beside spike edge and combines with shear film. An empirical relation is obtained in order to analyse the results of NPR that lies in range of 135140. For NPR of 50, the pressure distribution is unstable due to fluctuating movement in flow. Thus, the involvements of jet behavior influence the base pressure. For external flow the NPR results for spike ignoble pressure is higher than other NPR. This external flow is responsible for origin of oblique shock wave and creates a slip positions between jets and external flows [9].

\section{Aerodynamic thrust}

The specific impulse and average thrust considered for the optimization are- $3000 \mathrm{~s}$ and $2 \mathrm{~N}$ respectively. The value of compression thrust for the aerospike nozzle lies about 30-50\% around of thrust value. The value for internal expansion ratio is 1.26 that lies for extended flow for exhaust gases. The thrust performances for aerospike nozzle were measured for Mach number of 2.1 and at an altitude of $9.3 \mathrm{~km}$ [11]. The thrust vectors for conventional bell nozzles are influenced by movable gimbals or flaps. For slighter thrust classes, this mechanism can be performed injection of inferior flow indulge in prime flow for point thrusters. The thrust performance for this nozzle is studied by considering two cases i.e., one for the injected portion at $60 \%$ of short plug and other at $90 \%$ of condensed plug for which mass flow rate varies in range of $0.78-3.92 \%$ of prime flow [2]. For full length spike nozzle, the aerodynamic thrust for external flow is greater than those of spike forms lying above NPR of 50 for 2-D geometry variation. For external flow, the thrust increases for it that is similar to $25 \%$ of the point instances. In presence of external flow, the thrust efficiency is improved by $25 \%$ of spike instances under NPR of 50 for 2-D nozzle as shorter length results in load reserves. The thrust coefficients increases as of 1.6 at an NPR of 70 which increases with decreases in NPR below 70 for 2-D as well as cell based nozzles. At $50 \%$ of spike region, the thrust coefficient is lower in absence of flow than for the $25 \%$ of spike for NPR of 70 . The thrust coefficients become identical at $25 \%$ of spike for NPR above 70. However, the three cell nozzle has a higher performance as the configuration of it lays in a shape of clustered which also results in better reliability and safety considerations [9].

\section{E. Propulsive Characteristics}

The thrust for the aerospike nozzle is given by thrust equation as given below-

$$
\mathrm{F}=\dot{\mathrm{m}} \mathrm{V}_{\mathrm{e}}+\left(\mathrm{P}_{\mathrm{e}}-\mathrm{P}_{\mathrm{a}}\right) \mathrm{Ae}
$$

The specific impulse is given by-

$$
\mathrm{I}_{\mathrm{sp}}=\mathrm{F} /(\dot{\mathrm{m} g})
$$

$\mathrm{I}_{\mathrm{sp}}=\mathrm{F} /(\dot{\mathrm{mg}})$
Also, the specific thrust

$$
\mathrm{T}=\mathrm{F} / \dot{\mathrm{m}}_{\text {air }}
$$

defined by-

The specific thrust depends on variation in velocity of gas via engine component that is responsible for comparison of exit velocity and thrust. The thrust coefficient $\left(\mathrm{C}_{\mathrm{f}}\right)$ for the nozzle is given by-

$$
\mathrm{C}_{\mathrm{f}}=\mathrm{F} /\left(\mathrm{A}_{\mathrm{t}} \mathrm{P}_{\mathrm{c}}\right)
$$

This coefficient is responsible for the performance of the nozzle at altered conditions. The result for the configuration shows that the specific impulse increases for lesser equivalence ratio whereas the specific thrust decreases with decrease in equivalence ratio [10]. Specific impulse and specific thrust obtained for choked flow nozzle is about 4-6\% higher than those for open nozzle. As of increase in thrust, the momentum also increases for the nozzle and results in higher formation for choking condition. It is also noted that the operation between complete and shortened nozzle lies within $2 \%$ of overall area. The results for the characteristics velocity is more at the stagnation ratio when temperature is at its peak level [8]. 


\section{International Journal of Engineering Applied Sciences and Technology, 2021 \\ Vol. 5, Issue 11, ISSN No. 2455-2143, Pages 128-133 \\ Published Online March 2021 in IJEAST (http://www.ijeast.com)}

\section{CONCLUSION}

For design conditions at an altitude of $4210 \mathrm{~m}$ for $\eta_{\text {trun }}$ of $57 \%$ the thrust level obtained are $478 \mathrm{~N}$ and the spike truncation ratio of $75 \%$ the thrust level increases to 481.3 N. From these results it is clear that the performance of the nozzle decreases by $6 \%$ than the earlier estimation. Due to reduce in the pressure about $660 \mathrm{kPa}$ the thrust and mass flow rate reduces by $20 \%$ in all. The CFD characteristics considered for simulation and its design requirement as inlet temperature at $300 \mathrm{~K}$ and chamber pressure of $775 \mathrm{kPa}$. The divergent results for CFD and method of characteristics steps down the pressure for throat region for which the flow angle is idealized and characteristics line is responsible for the breakage of codes. At downstream of injected interface, a low pressure region is generated by expansion of fluid and a higher region is formed by extension of bow shockwaves. Due to this the local pressure value increases which results in amplification of thrust created for secondary flow. At $50 \%$ of spike region, the thrust coefficient is lower in absence of flow than for the $25 \%$ of spike for NPR of 70. Specific impulse and specific thrust obtained for choked flow nozzle is about 4-6\% higher than those for open nozzle. As of increase in thrust, the momentum also increases for the nozzle and results in higher formation for choking condition. It is also noted that the operation between complete and shortened nozzle lies within $2 \%$ of overall area.

\section{REFERENCE}

[1] Shannon D. Eilers, Matthew D. Wilson and Stephen A. Whitmore. (2010). "Analytical and Experimental Evaluation of Aerodynamic Thrust Vectoring on an Aerospike Nozzle", 46 ${ }^{\text {th }}$ AIAA/ASME/SAE/ASEE Joint Propulsion Conference \& Exhibit 25 - 28.

[2] M. Propst, J. Sieder, C. Bach and M. Tajmai. (2014). " NUMERICAL ANALYSIS ON AN AERODYNAMICALLY THRUSTVECTORED AEROSPIKE NOZZLE," Conference paper.

[3] Ajith S, Anoovendhan S, Meghana Raj, Ramya S, Tharikaa R, Vivek S, V.R Sanal Kumar. (2016). " Performance Evaluation of Aerospike Nozzles for Lucrative Thrust Vector Control ," Propulsion and Energy Forum, July 25-27.

[4] Prasanth P Nair, Abhilash Suryan and Heuy Dong Kim. (2017). "Computational Study of Performance Characteristics for Truncated Conical Aerospike Nozzles",Journal of Thermal Science, Vol.26, No.6, pp. 483-489.

[5] Md Saquib Reza1, Konark Arora. (2017). "Contour Design of Aerospike Nozzle and Comparison of Performance", International Conference on Infocom Technologies and Unmanned Systems (ICTUS), Dec. 1820.
[6] O Dumitrescu, B Gherman and Valeriu Dragan. (2017). " Study of full and truncated aerospike nozzles on performances at different working conditions", IOP Conf. Series: Materials Science and Engineering.

[7] Douglas A. Schwer, Robert Kelso and Christopher M. Brophy. (2018). "Pressure Characteristics of an Aerospike Nozzle in a Rotating Detonation Engine", AIAA Propulsion and Energy Forum, July 9-11.

[8] Nobuhiro Kurita1, Nicolas Jourdaine, Nobuyuki Tsuboi, and Kohei Ozawa. (2020). "Three-Dimensional Numerical Simulation on Hydrogen/Air Rotating Detonation Engine with Aerospike Nozzle: Effects of Nozzle Geometries", AIAA SciTech Forum, 6-10.

[9] Hidemi Takahashi, Sadatake Tomioka, Takeo Tomita, and Noboru Sakuranaka (2015). "Aerodynamic Characterization of Linear Aerospike Nozzles in OffDesign Flight Conditions", JOURNAL OF PROPULSION AND POWER Vol. 31, No. 1.

[10] K. Schomberg and J. Olsen. (2012). "Altitude compensation in expansion deflection nozzles," In Proceedings of the 18th Australian Fluid Mechanics Conference, Launceston, Australia.

[11] Nobuyuki Tsuboi1, Yuzi Kawakami, Kyushu Institute of Technology, Kitakyushu, Ryosuke Ezure , A. Koichi Hayashi, and Eisuke Yamada. (2011). 49th AIAA Aerospace Sciences Meeting including the New Horizons Forum and Aerospace Exposition.

[12] Prasanth P. Naira , Abhilash Suryana, Heuy Dong Kim. (2019). Propulsion and Power Research , 8(2):108-120. 